\title{
A dynamic clustering algorithm for downlink CoMP systems with multiple antenna UEs
}

\author{
Paolo Baracca ${ }^{1 *}$, Federico Boccardi ${ }^{2}$ and Nevio Benvenuto ${ }^{3}$
}

\begin{abstract}
Coordinated multi-point (COMP) schemes have been widely studied in the recent years to tackle inter-cell interference. In practice, latency and throughput constraints on the backhaul allow the organization of only small clusters of base stations (BSs) where joint processing (JP) can be implemented. In this work, we focus on downlink CoMP-JP with multiple antenna user equipments (UEs) where the additional degrees of freedom are used to suppress the residual interference by using an interference rejection combiner (IRC) and allow a multi-stream transmission. The main contribution of this paper is the development of a novel dynamic BS clustering algorithm with corresponding UE scheduling. In particular, we first define a set of candidate BS clusters depending on long-term channel conditions. Then, in each time block, we develop a resource allocation scheme where: (a) for each candidate BS cluster, with corresponding scheduled UEs, a weighted sum rate is estimated and then (b) we select the set of non-overlapping BS clusters that maximizes the downlink system weighted sum rate. Numerical results show that much higher rates are achieved when UEs are equipped with multiple antennas and dynamic BS clustering is used.
\end{abstract}

Keywords: Resource allocation and interference management; MIMO systems; Cellular technology

\section{Introduction}

Coordination among base stations (BSs) has been widely studied in the recent years to tackle inter-cell interference which strongly limits the rates achieved in cellular systems, in particular by the user equipments (UEs) at the cell-edge [1]. Supported by the first results promising huge gains with respect to the baseline non-cooperative system [2], a lot of attention has been paid to the topic both in the academia $[3,4]$ and in the industry $[5,6]$. These techniques, known in the industry as coordinated multipoint (CoMP), are classified into (a) coordinated scheduling/beamforming $(\mathrm{CS} / \mathrm{CB})$, which requires channel state information (CSI) but no data sharing among the BSs, and (b) joint processing (JP), which requires both CSI and data sharing among the BSs. This paper focuses on downlink CoMP-JP, where BSs jointly serve the scheduled UEs by sharing the data to be sent. Although CoMP-JP is a very promising technique, many issues make its implementation still challenging. First, CSI at the transmitter may be unreliable because of noise on channel estimation in time

*Correspondence: paolo.baracca@alcatel-lucent.com

1 Bell Labs, Alcatel-Lucent, Lorenzstrasse 10, Stuttgart 70435, Germany

Full list of author information is available at the end of the article division duplex (TDD) systems and limited bandwidth available for feedback in frequency division duplex systems. Then, sharing UE data among all the BSs is generally limited by throughput and delay constraints in the backhaul infrastructure. A possible approach to deal with backhaul throughput constraints relies on partial sharing of UE data among the BSs, i.e., a BS serving a certain UE may have only a partial knowledge of the data to be sent toward that UE $[7,8]$. Although the promising results achieved under idealistic assumptions, partial UE data sharing has not found application in real systems mainly due to its complexity. To deal with limited throughput backhaul, most of the works in the literature focus on the simpler clustering approach where the BSs are organized in clusters and joint processing is applied within each cluster by sharing the whole data to be sent among all the BSs of the cluster. However, even if intra-cluster interference is mitigated by using CoMP schemes within each cluster, UEs at the cluster border suffer strong inter-cluster interference (ICI). Many clustering schemes have been developed in the literature to deal with ICI. In [9], static clustering with block diagonalization is considered and precoders are designed in each cluster by nullifying the interference towards UEs of neighboring clusters close to the border.

\section{Springer}

(c) 2014 Baracca et al: licensee Springer. This is an Open Access article distributed under the terms of the Creative Commons Attribution License (http://creativecommons.org/licenses/by/2.0), which permits unrestricted use, distribution, and reproduction in any medium, provided the original work is properly credited. 
A more flexible solution is obtained with dynamic clustering $[10,11]$ where the set of clusters changes over time by adapting to the network conditions. In particular, in [10], a greedy algorithm is developed where, for each cluster, the first $\mathrm{BS}$ is selected randomly to guarantee fairness, while the remaining BSs are selected by maximizing the cluster sum rate. In [11] instead, based on the long-term channel conditions, it is defined a set of candidate clusterings and then in each time block is selected the most suitable one. In [12], selected clusters maximize the increase of the achievable UE rate, whereas in $[12,13]$, they minimize the interference power. In [14], a BS negotiation algorithm is used for cluster formation within a given cluster size. In [15], active clusters are selected by minimizing an overall cost function which depends on the UE average received power. A framework for feedback and backhaul overhead reduction is developed in [16] where each UE feeds back CSI only to a subset of BSs, and UEs associated to the same subset are grouped together. In [17], a greedy UE scheduling algorithm with overlapping clusters is proposed where precoders are designed by considering the layered virtual signal to interference plus noise ratio (SINR) criterion [18]. In [19], an iterative algorithm is proposed to jointly optimize beamforming and clustering in heterogeneous networks.

However, most of the works on dynamic clustering ([10-13,16,17]) assume that UEs are equipped with only one antenna, although the Long Term Evolution (LTE) Advanced standard developed by the 3rd Generation Partnership Project (3GPP) considers that UEs may be equipped with up to eight antennas [20]. Although this number seems a bit optimistic for current mobile devices, the technological innovation may allow in the near-future manufacturing smartphones or tablets with numerous antennas and hence much more attention should be paid to the study of CoMP schemes with multiple antenna UEs $[21,22]$. Therefore, in this work, we consider downlink CoMP-JP with a constraint on the maximum cluster size and propose a novel dynamic BS clustering and UE scheduling algorithm by explicitly considering that UEs are equipped with multiple antennas. In our proposal, UEs exploit these additional degrees of freedom by implementing interference rejection combiner (IRC) [23] to partially suppress ICI and being served by means of a multi-stream transmission. Moreover, differently from many works on dynamic clustering where UE selection is not considered and a simple round robin scheduler is implemented ([10,12-14,16,19]), here, we assume UE scheduling as a part of the optimization. In our approach, we first define a set of candidate BS clusters depending on long-term properties of the channels. Then, in each time block, the proposed algorithm follows a two-step procedure: (a) a weighted sum rate is estimated for each candidate cluster by performing UE selection, precoding design, power allocation, and transmission rank selection and then (b) the central unit $(\mathrm{CU})$ coordinating all the BSs schedules the set of non-overlapping candidate clusters that maximizes the system weighted sum rate under the assumption of perfect successive interference cancellation (SIC) with IRC at each UE.

For a performance comparison, we use the effective achievable rate at UEs, by assuming that CSI is perfectly known at the receiver. In particular, we evaluate the achievable rate of the proposed solution in a LTE-TDD scenario and compare it against a baseline single-cell processing (SCP) scheme and two static clustering schemes, where clusters do not dynamically adapt to the network conditions. Numerical results show that the achievable rates strongly increase with the number of UE antennas. Moreover, as with CoMP part of the interference is managed at the transmit side, multi-stream transmission is more effective with the proposed scheme than with SCP. However, as most of the gain is due to the interference suppression capability of the IRC, the relative gain achieved by the proposed scheme with respect to SCP decreases by increasing the number of UE antennas. Finally, a further decrease of this gain is observed when imperfect CSI is considered at BSs.

Notation. We use $(\cdot)^{T}$ to denote transpose and $(\cdot)^{H}$ conjugate transpose. $\mathbf{0}_{N \times M}$ denotes the matrix of size $N \times M$ with all zero entries, $\boldsymbol{I}_{N}$ the identity matrix of $\operatorname{size} N, \operatorname{tr}(\boldsymbol{X})$ the trace of matrix $\boldsymbol{X}, \operatorname{det}(\boldsymbol{X})$ the determinant of matrix $\boldsymbol{X}$, $\operatorname{vec}(\boldsymbol{X})$ the vectorization of $\boldsymbol{X},\|\boldsymbol{X}\|$ the Frobenius norm of $\boldsymbol{X},[\boldsymbol{X}]_{n, m}$ the entry on row $n$ and column $m$ of $\boldsymbol{X},[\boldsymbol{X}]_{,, m}$ the $m$ th column of $X$, and diag $(\boldsymbol{x})$ the diagonal matrix with the entries of vector $\boldsymbol{x}$ on the diagonal. Expectation is denoted by $\mathbb{E}[\cdot]$.

\section{System model}

We consider a TDD system where a set of BSs $\mathcal{J}=$ $\{1,2, \ldots, J\}$, each equipped with $M$ antennas, is serving a set of UEs $\mathcal{K}=\{1,2, \ldots, K\}$, each equipped with $N$ antennas, with $K>J M$. As the overall number of transmitting antennas is not sufficient to serve all the UEs at the same time, UE scheduling is part of the optimization problem. We assume a block fading channel model and denote with $\boldsymbol{H}_{k, j}(t), t=0,1, \ldots, T-1$, the multiple-input multipleoutput (MIMO) channel matrix of size $N \times M$ between BS $j$ and UE $k$ in block $t$. We consider that the entries of matrix $\boldsymbol{H}_{k, j}(t)$ are identically distributed zero-mean complex Gaussian random variables, i.e., $\left[\boldsymbol{H}_{k, j}(t)\right]_{n, m} \sim$ $\mathcal{C N}\left(0, \sigma_{k, j}^{2}\right)$, for $n=0,1, \ldots, N-1$ and $m=0,1, \ldots, M-$ 1 , where $\sigma_{k, j}^{2}$ represents the large scale fading between BS $j$ and UE $k$, which depends on path loss and shadowing. We assume that the statistical description of the channels does not change for all the $T$ blocks, whereas fast fading realizations are independent among different blocks. Then, 
we denote with $\boldsymbol{\Sigma}_{k, j}=\mathbb{E}\left[\operatorname{vec}\left(\boldsymbol{H}_{k, j}(t)\right) \operatorname{vec}\left(\boldsymbol{H}_{k, j}(t)\right)^{H}\right]$ the covariance matrix of the channel matrix $\boldsymbol{H}_{k, j}(t)$. We indicate with $L_{E}$ the number of resource elements, i.e., time slots, forming a block. Note that the block fading model considered in this work can be adapted to represent a more realistic channel which changes continuously both in time and in frequency by suitably selecting the number of resource elements in each block. In fact, by denoting with $W_{C}$ and $T_{C}$ the coherence bandwidth and time of the channel, respectively, we have $L_{E}=W_{C} T_{C}$.

We assume that the BSs are coordinated by a $\mathrm{CU}$, and the backhaul links have zero latency and are error-free. Each block is organized in three phases: (a) in the first phase, all the UEs send pilot sequences to allow channel estimation at BSs, (b) in the second phase, BS clustering, UE scheduling, beamforming design, transmission rank selection, and power optimization are performed by the $\mathrm{CU}$, and finally, (c) in the third phase, the BSs perform data transmission toward the set of scheduled UEs.

For the sake of clarity, we report in Table 1 the list of important symbols used in this work.

\subsection{First phase: uplink pilot transmission}

The first $L_{T}$ resource elements of each block are allocated to the uplink pilot transmission performed by the UEs. We assume that orthogonal sequences, each of length $L_{T}$, are employed by the UEs; thus, interference on channel estimation is avoided at BSs: in the considered scenario, this

\begin{tabular}{|c|c|}
\hline Symbol & Definition \\
\hline $\mathcal{J}$ & Set of BSs, each equipped with $M$ antennas \\
\hline $\mathcal{K}$ & Set of UEs, each equipped with $N$ antennas \\
\hline $\mathcal{C}$ & Set of integers, each identifying a candidate BS clusters \\
\hline $\mathcal{J}_{c}$ & Set of BSs forming candidate BS cluster $c, c \in \mathcal{C}$ \\
\hline $\mathcal{S}$ & Set of scheduled UEs \\
\hline $\mathcal{U}_{c}$ & Set of UEs corresponding to BSs in $\mathcal{J}_{C}$ (see (12)) \\
\hline $\mathcal{S}_{c}$ & Set of scheduled UEs corresponding to BSs in $\mathcal{J}_{c}$ (see (16a)) \\
\hline $\boldsymbol{H}_{k j}$ & Channel between BS $j$ and UE $k$ \\
\hline$\hat{\boldsymbol{H}}_{k j}$ & Estimated channel between BS $j$ and UE $k$ \\
\hline$\hat{\boldsymbol{H}}_{k}^{(c)}$ & Estimated channel between BSs in $\mathcal{J}_{c}$ and UE $k$ \\
\hline $\boldsymbol{G}_{k, j}$ & Precoding matrix used by BS $j$ to serve UE $k$ \\
\hline $\boldsymbol{G}_{k}^{(c)}$ & Precoding matrix used by BSs in $\mathcal{J}_{c}$ to serve UE $k$ \\
\hline$l_{k}$ & Transmission rank allocated to UE $k$ \\
\hline $\boldsymbol{P}_{k}$ & Power vector allocated to UE $k$ \\
\hline$\hat{R}^{(c)}$ & Estimated weighted sum rate achieved by BSs in $\mathcal{J}_{c}$ \\
\hline$\hat{R}_{k}$ & Estimated rate achieved by UE $k$ \\
\hline$R_{k}$ & Effective rate achieved by UE $k$ \\
\hline
\end{tabular}

is achieved by imposing a minimum length of the training sequence of $L_{T} \geq N K$. By denoting with $P^{(\mathrm{UE})}$ the maximum power available at each UE and $\sigma_{n}^{2}$ the thermal noise power, under the assumption of perfect reciprocity, BS $j$ estimates the channel matrix $\boldsymbol{H}_{k, j}(t)$ connecting UE $k$ to itself from the observation

$$
\begin{aligned}
o_{k, j, n, m}(t)= & {\left[\boldsymbol{H}_{k, j}(t)\right]_{n, m}+\eta_{k, j, n, m}(t), } \\
& n=0,1, \ldots, N-1, m=0,1, \ldots, M-1,
\end{aligned}
$$

where $\eta_{k, j, n, m}(t) \sim \mathcal{C N}\left(0, \frac{N \sigma_{n}^{2}}{L_{T} P^{(\mathrm{UE})}}\right)$. By assuming that BS $j$ knows the covariance matrix $\boldsymbol{\Sigma}_{k, j}$, the minimum mean square error (MMSE) estimate $\hat{\boldsymbol{H}}_{k, j}(t)$ of $\boldsymbol{H}_{k, j}(t)$ given the observation (1) can be written as ([24], [Ch. 10])

$$
\begin{aligned}
\operatorname{vec}\left(\hat{\boldsymbol{H}}_{k, j}(t)\right)= & \boldsymbol{\Sigma}_{k, j}\left(\boldsymbol{\Sigma}_{k, j}+\frac{N \sigma_{n}^{2}}{L_{T} P^{(\mathrm{UE})}} \boldsymbol{I}_{M N}\right)^{-1} \\
& \times\left(\operatorname{vec}\left(\boldsymbol{H}_{k, j}(t)\right)+\operatorname{vec}\left(\boldsymbol{\eta}_{k, j}(t)\right)\right),
\end{aligned}
$$

where $\left[\boldsymbol{\eta}_{k, j}(t)\right]_{n, m}=\eta_{k, j, n, m}(t)$.

Note that in the case of uncorrelated channels, i.e., when $\boldsymbol{\Sigma}_{k, j}=\boldsymbol{I}_{M N}$, the expression in (2) turns out to be

$$
\hat{\boldsymbol{H}}_{k, j}(t)=\frac{1}{1+\frac{N \sigma_{n}^{2}}{L_{T} P^{(\mathrm{UE})} \sigma_{k, j}^{2}}}\left(\boldsymbol{H}_{k, j}(t)+\boldsymbol{\eta}_{k, j}(t)\right) \text {. }
$$

\subsection{Second phase: resource allocation at the $\mathrm{CU}$}

After uplink pilot transmission, each BS $j$ forwards the channel estimates $\hat{\boldsymbol{H}}_{k, j}(t), k \in \mathcal{K}$, to the CU, which, in turn, organizes BSs in clusters and schedules in each time block $t$ a subset $\mathcal{S}(t) \subseteq \mathcal{K}$ of UEs.

In this work, we consider dynamic multi-stream transmission and we denote with $l_{k}(t)$ the transmission rank allocated to UE $k$ in block $t$, i.e., the number of streams sent toward UE $k$. Let us denote with $\boldsymbol{G}_{k, j}(t)$ the $M \times l_{k}(t)$ beamforming matrix used by BS $j$ to serve UE $k$ and with $\boldsymbol{P}_{k}(t)=\left[P_{k, 0}(t), P_{k, 1}(t), \ldots, P_{k, l_{k}(t)-1}(t)\right]$ the power allocation vector for UE $k$.

A basic approach to BS clustering is static, i.e., BS clusters are fixed and do not change over time. For example, in the hexagonal setup with seven sites and three BSs per site, reported in Figure 1, typical standard configurations are the following:

- Single cell processing (SCP), where no cooperation is allowed among the BSs and each UE is served by its anchor BS, i.e., the BS characterized by the highest signal to noise ratio (SNR) (baseline scheme).

- Intra-site cooperation (ISC), where seven clusters are constructed, each one composed by three co-located 


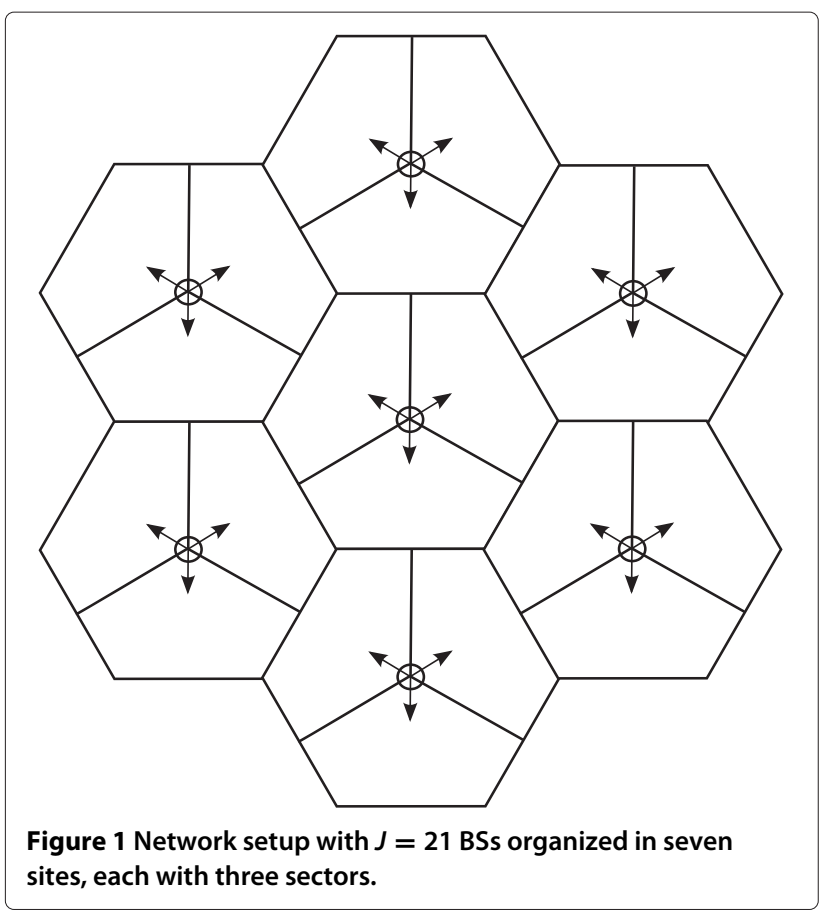

BSs (see Figure 2), and each UE is served by the best site in terms of average SNR.

- Static clustering (SC), where still seven static clusters are constructed, but with cooperation allowed among three BSs of three different sites as shown in Figure 3.

Here, $\mathcal{C}=\{1,2, \ldots,|\mathcal{C}|\}$ denotes the set of integers, each identifying a candidate BS cluster, while $\mathcal{J}_{c}(t) \subseteq$ $\mathcal{J}$ is the $c$-th candidate BS cluster and $\mathcal{S}_{c}(t) \subseteq \mathcal{K}$ the

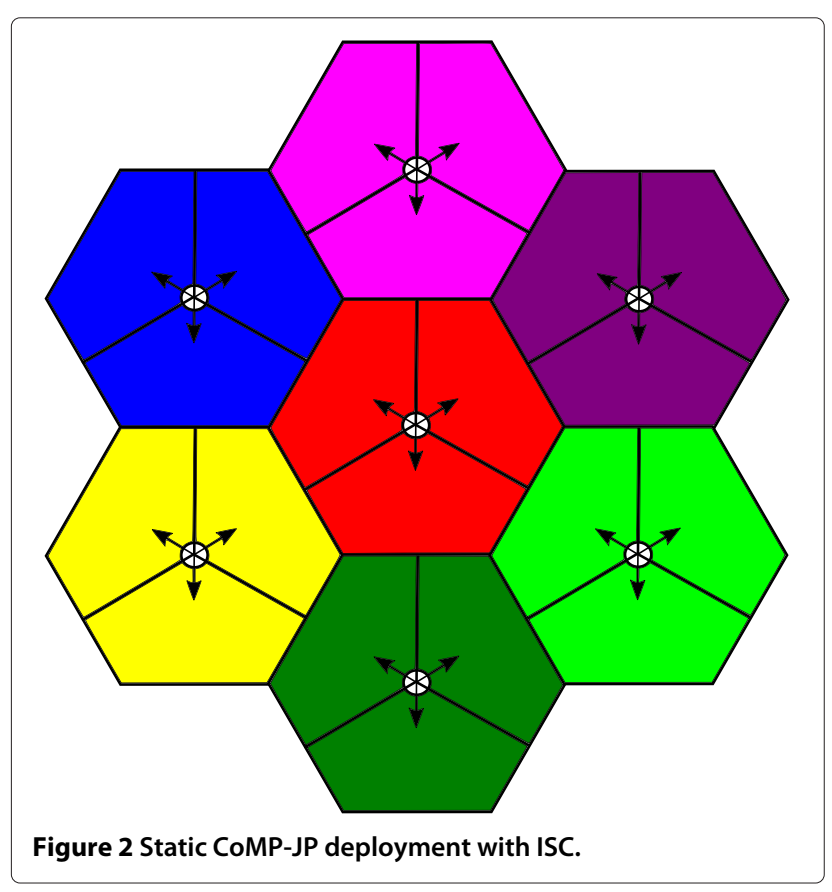

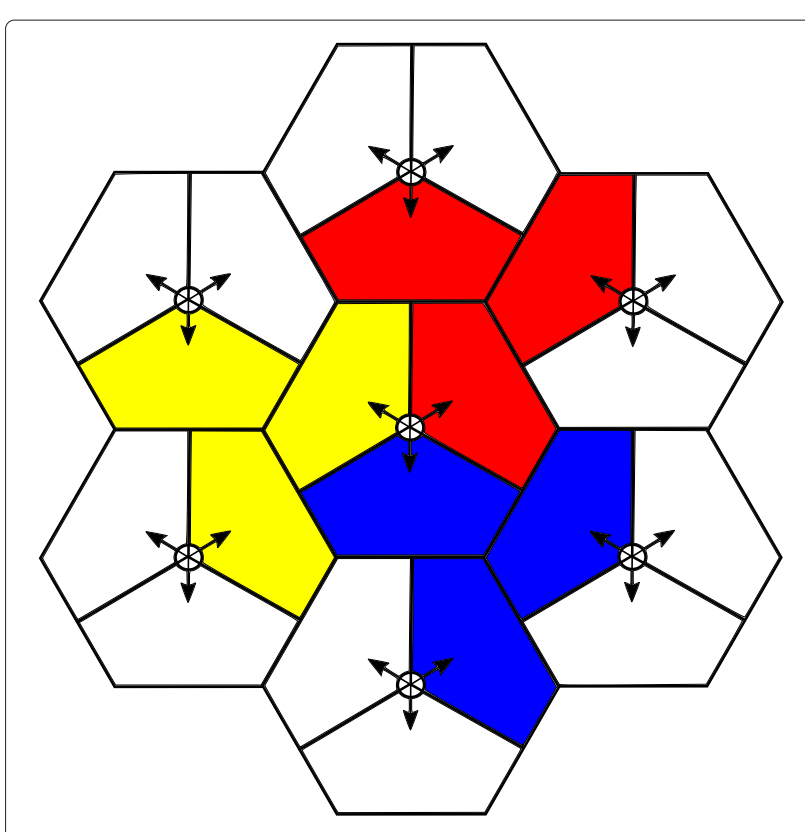

Figure 3 Static CoMP-JP deployment with SC.

corresponding set of scheduled UEs. For complexity reasons, in this work, we consider non-overlapping clusters: hence, in each time block $t$, the set $\mathcal{J}$ of BSs is partitioned into non-overlapping clusters and no UE can be served in the same time block by two different BS clusters. Although a solution with overlapping clusters would provide higher rates, it would be much more challenging in terms of computational complexity, in particular when the number of BSs $J$ managed by the CU is high.

We denote with $\hat{\boldsymbol{H}}_{k}^{(c)}(t)$ the matrix of size $N \times M\left|\mathcal{J}_{c}(t)\right|$ collecting the MIMO channel estimated by BSs in cluster $\mathcal{J}_{c}(t)$ toward UE $k$ and with $\boldsymbol{G}_{k}^{(c)}(t)$ the corresponding precoding matrix of size $M\left|\mathcal{J}_{c}(t)\right| \times l_{k}(t)$, with $\left\|\left[G_{k}^{(c)}(t)\right]_{, l}\right\|^{2}=1, l=0,1, \ldots, l_{k}(t)-1$. Due to the imperfect CSI at BSs, the signal received by UE $k$ is modeled at the CU as

$$
\begin{aligned}
\hat{\boldsymbol{r}}_{k}^{(c)}(t)= & \hat{\boldsymbol{H}}_{k}^{(c)}(t) \boldsymbol{G}_{k}^{(c)}(t) \boldsymbol{s}_{k}(t)+ \\
& \sum_{m \in \mathcal{S}_{c}(t) \backslash\{k\}} \hat{\boldsymbol{H}}_{k}^{(c)}(t) \boldsymbol{G}_{m}^{(c)}(t) \boldsymbol{s}_{m}(t)+\boldsymbol{n}_{k}(t)+\hat{\boldsymbol{i}}_{k}^{(c)}(t),
\end{aligned}
$$

where $\boldsymbol{s}_{k}(t) \sim \mathcal{C N}\left(\mathbf{0}_{l_{k}(t) \times 1}, \operatorname{diag}\left(\boldsymbol{P}_{k}(t)\right)\right)$ is the data symbol vector transmitted toward UE $k, \boldsymbol{n}_{k}(t) \sim$ $\mathcal{C N}\left(\mathbf{0}_{N \times 1}, \sigma_{n}^{2} \boldsymbol{I}_{N}\right)$ is the thermal noise at the UE antennas and $\hat{\boldsymbol{i}}_{k}^{(c)}(t)$ is the estimate of the ICI suffered by UE $k$. Note that the exact value of $\hat{\boldsymbol{i}}_{k}^{(c)}$ depends on the beamformers used by other clusters to serve their own UEs. By indicating with $P^{(\mathrm{BS})}$ the power available at each BS, 
beamformers are designed by assuming per-BS power constraints, i.e.,

$$
\sum_{k \in \mathcal{S}_{c}(t)} \operatorname{tr}\left(\boldsymbol{G}_{k, j}^{H}(t) \boldsymbol{G}_{k, j}(t) \operatorname{diag}\left(\boldsymbol{P}_{k}(t)\right)\right) \leq P^{(\mathrm{BS})}, j \in \mathcal{J}_{c}(t) .
$$

In this work, we make the following assumptions regarding power allocation and beamforming design.

- Equal power is allocated to the streams sent toward the UEs scheduled within the same cluster, i.e., $P_{k, l}(t)=P^{(c)}(t), k \in \mathcal{S}_{c}(t), l=0,1, \ldots, l_{k}(t)-1$, where $P^{(c)}(t)$ can be analytically computed from (5) as

$$
P^{(c)}(t)=\frac{P^{(\mathrm{BS})}}{\max _{j \in \mathcal{J}_{c}(t)} \sum_{k \in \mathcal{S}_{c}(t)} \sum_{l=0}^{l_{k}^{(t)-1}}\left\|\left[\boldsymbol{G}_{k, j}(t)\right]_{, l, l}\right\|^{2}} .
$$

- Beamformers are designed by using the multi-user eigenmode transmission (MET) scheme [25], where the precoding matrix used to serve UE $k$ is optimized with the aim of nullifying the interference toward the eigenmodes selected for the co-scheduled UEs $m \in \mathcal{S}_{c}(t) \backslash\{k\}$. In detail, let $\hat{\boldsymbol{H}}_{k}^{(c)}(t)=\hat{\boldsymbol{U}}_{k}^{(c)}(t) \hat{\boldsymbol{\Sigma}}_{k}^{(c)}$ $(t) \hat{\boldsymbol{V}}_{k}^{(c) H}(t)$ be the singular value decomposition (SVD) of matrix $\hat{\boldsymbol{H}}_{k}^{(c)}(t)$, where the eigenvalues in $\hat{\boldsymbol{\Sigma}}_{k}^{(c)}(t)$ are arranged so that the ones selected for transmission toward UE $k$ appear in the leftmost columns. By defining matrix

$$
\begin{aligned}
& \hat{\boldsymbol{\Gamma}}_{k}^{(c)}(t)= \\
& {\left[\left[\hat{\boldsymbol{\Sigma}}_{k}^{(c)}(t)\right]_{0,0}\left[\hat{\boldsymbol{V}}_{k}^{(c)}(t)\right]_{, 0,0},\left[\hat{\boldsymbol{\Sigma}}_{k}^{(c)}(t)\right]_{1,1}\left[\hat{\boldsymbol{V}}_{k}^{(c)}(t)\right]_{\cdot, 1},\right.} \\
& \left.\ldots,\left[\hat{\boldsymbol{\Sigma}}_{k}^{(c)}(t)\right]_{l_{k}(t)-1, l_{k}(t)-1}\left[\hat{\boldsymbol{V}}_{k}^{(c)}(t)\right]_{\cdot, l_{k}(t)-1}\right]^{H},
\end{aligned}
$$

precoding matrix used to serve UE $k$ satisfies constraints

$$
\hat{\boldsymbol{\Gamma}}_{m}^{(c)}(t) \hat{\boldsymbol{G}}_{k}^{(c)}(t)=\mathbf{0}_{l_{m}(t) \times l_{k}(t)}, \quad k \neq m .
$$

Note that MET has been proven to outperform in a MIMO broadcast channel other linear precoding schemes such as block diagonlization [25], whereas the assumption of equal power allocation among the scheduled streams reduces the computational complexity and is asymptotically optimal at high SNR.

The main contribution of this work, described in Section 3, is a practical algorithm for dynamic BS clustering and corresponding UE scheduling developed in the considered setup with multiple antenna BSs, with equal power allocation and MET, that transmit toward multiple antenna UEs. Moreover, we recall that at UEs, the multiple receive antennas are used to perform IRC with SIC ([26], Ch. 10); in fact, while the rank $l_{k}(t)$ allocated to UE $k$ is given by the number of columns of the precoder, the remaining degrees of freedom at UE are used to partially suppress the residual ICI. Note that IRC both minimizes the mean square error and maximizes the SINR at the detection point [23].

\subsection{Third phase: downlink data transmission}

BS clusters serve the scheduled UEs by using the $L_{E}-L_{T}$ resource elements still available in block $t$. By defining matrix $\boldsymbol{G}_{k}(t)=\left[\boldsymbol{G}_{k, 1}^{T}(t), \boldsymbol{G}_{k, 2}^{T}(t), \ldots, \boldsymbol{G}_{k, J}^{T}(t)\right]^{T}$ and matrix $\boldsymbol{H}_{k}(t)=\left[\boldsymbol{H}_{k, 1}(t), \boldsymbol{H}_{k, 2}(t), \ldots, \boldsymbol{H}_{k, J}(t)\right]$, the signal received by UE $k$ can be written as

$$
\boldsymbol{r}_{k}(t)=\boldsymbol{H}_{k}(t) \boldsymbol{G}_{k}(t) \boldsymbol{s}_{k}(t)+\sum_{m \in \mathcal{S}(t) \backslash\{k\}} \boldsymbol{H}_{k}(t) \boldsymbol{G}_{m}(t) \boldsymbol{s}_{m}(t)+\boldsymbol{n}_{k}(t) .
$$

\section{Dynamic clustering algorithm}

In this section, we drop the block index $t$ for the sake of clarity. With respect to UE $k$, let us define the function $f_{k}: \mathcal{J} \rightarrow \mathcal{J}$, which orders the BSs on the basis of the large scale fading component of the channel, i.e., $\sigma_{k, f_{k}\left(c_{1}\right)}^{2}>$ $\sigma_{k, f_{k}\left(c_{2}\right)}^{2}$ if $c_{1}<c_{2}$. Then, we indicate with $\mathcal{J}_{k}^{(u)}$ the cluster of the $u \mathrm{BSs}$ with the strongest average channel toward UE $k$, i.e.,

$$
\mathcal{J}_{k}^{(u)}=\left\{f_{k}(1), f_{k}(2), \ldots, f_{k}(u)\right\} .
$$

Hence, $f_{k}(1)$ is the anchor BS for UE $k$.

In a network with $J$ BSs and a maximum cluster size of $J_{\mathrm{MAX}}$, the number of possible BS clusters that can be constructed to serve a given UE is

$$
\sum_{j=1}^{J_{\mathrm{MAX}}}\left(\begin{array}{l}
J \\
j
\end{array}\right)
$$

which rapidly increases with $J$. However, as most of the interference at each UE comes from the closest BSs, we can limit the number of candidate BS clusters. Hence, we assume that set $\mathcal{C}$ identifies all and only the sets $\mathcal{J}_{k}^{(u)}$ whose size is not bigger than $J_{\mathrm{MAX}}$. As an example, selecting $J_{\mathrm{MAX}}=3$, set $\mathcal{C}$ identifies three candidate clusters for each UE $k$ : (i) the first cluster includes only its anchor BS, $\mathcal{J}_{k}^{(1)}$, (ii) the second cluster is composed of the two closest BSs, $\mathcal{J}_{k}^{(2)}$, and (iii) the third cluster is composed of the three closest BSs, $\mathcal{J}_{k}^{(3)}$. Note that, as different UEs often have the same candidate clusters, the cardinality of set $\mathcal{C}$ turns out to be much lower than $K J_{\mathrm{MAX}}$. The considered assumption yields an important saving in terms of computational complexity by strongly limiting the number of 
candidate clusters with respect to (11): this complexity saving is evaluated in Section 4 for a typical LTE scenario.

Now, clusters $\mathcal{J}_{k}^{(u)}$, with $u \leq J_{\text {MAX }}$, are ordered by an integer index $c$, and for each cluster $\mathcal{J}_{c}, c \in \mathcal{C}$, we define the corresponding set $\mathcal{U}_{c}$ of UEs that can be scheduled for reception, which is formed by the UEs whose anchor BS belongs to $\mathcal{J}_{c}$, i.e.,

$$
\mathcal{U}_{c}=\left\{k \in \mathcal{K}: f_{k}(1) \in \mathcal{J}_{c}\right\} .
$$

We highlight that (12) allows BSs in cluster $\mathcal{J}_{c}$ to serve all the UEs in its coverage area, even UEs close to the border. Although a different choice could be taken for instance by forcing clusters to serve only the UEs far away from the border, it has been shown in [27] that this alternative choice provides worse performance than (12) when a huge network is considered and fairness among the UEs is taken into account.

In this work, we propose an algorithm for dynamic BS clustering and UE scheduling at the $\mathrm{CU}$ which follows a two-step procedure.

1. For each candidate BS cluster $\mathcal{J}_{c}$, we estimate the weighted sum rate $\hat{R}^{(c)}$ by selecting a suitable subset of UEs $\mathcal{S}_{c} \subseteq \mathcal{U}_{c}$, designing precoders, selecting transmission ranks, and allocating powers.

2. After computing the weighted sum rate $\hat{R}^{(c)}$ for all the candidate BS clusters in $\mathcal{C}$, the $\mathrm{CU}$ schedules a set of non-overlapping BS clusters, where each BS belongs to at most one cluster.

Moreover, based on (12), we observe that UE $k$ can be selected only by candidate clusters that include its anchor BS $f_{k}(1)$. Hence, if we enforce a non-overlapping solution, each UE is never scheduled by two different non-overlapping clusters in the same block. However, we highlight that the proposed dynamic solution allows the flexibility of scheduling a given UE in different clusters across successive blocks.

In the rest of this section, we describe more in detail the above two main steps of the algorithm. We stress that the candidate cluster selection, i.e., the construction of set $\mathcal{C}$ depends on the large scale fading: hence, in our model, it should be performed only every $T$ blocks. On the other hand, the two-step algorithm described above follows a fast fading time-scale and therefore must be implemented in each block.

In Figure 4, we report a flow chart which summarizes the proposed system configuration.

\subsection{Cluster weighted sum rate estimation}

The CU estimates the weighted sum rate $\hat{R}^{(c)}$ that can be achieved by candidate cluster $\mathcal{J}_{c}$ by modeling the signal received by UE $k \in \mathcal{U}_{c}$ as in (4). Besides the errors due to the imperfect CSI (2), the estimated ICI $\hat{\boldsymbol{i}}_{k}^{(c)}$ depends on the precoders used by the other clusters scheduled by the $\mathrm{CU}$. In our framework, as the CU schedules the clusters after estimating the weighted sum rate achievable in each candidate, we simply assume $\hat{\boldsymbol{i}}_{k}^{(c)} \sim \mathcal{C N}\left(\mathbf{0}_{N \times 1}, \xi_{k}^{(c)} \boldsymbol{I}_{N}\right)$ with

$$
\xi_{k}^{(c)}=P^{(\mathrm{BS})} \sum_{j \in \mathcal{J} \backslash \mathcal{J}_{c}} \sigma_{k, j}^{2} .
$$

Note that (13) represents the average ICI power at the UE $k$ when all the BSs outside cluster $c$ are transmitting at full power ([28], (2)), and for each candidate cluster allows the computation of $\hat{R}^{(c)}$ independently of the other candidates.

From (4), we introduce the interference plus noise covariance matrix

$$
\begin{aligned}
\hat{\boldsymbol{\Psi}}_{k}^{(c)}= & \left(\sigma_{n}^{2}+\xi_{k}^{(c)}\right) \boldsymbol{I}_{N}+ \\
& \sum_{m \in \mathcal{S}_{c} \backslash\{k\}} \hat{\boldsymbol{H}}_{k}^{(c)} \boldsymbol{G}_{m}^{(c)} \operatorname{diag}\left(\boldsymbol{P}_{m}\right) \boldsymbol{G}_{m}^{(c) H} \hat{\boldsymbol{H}}_{k}^{(c) H} .
\end{aligned}
$$

By using (4) and (14), we can write the estimate at the CU of the rate $\hat{R}_{k}$ achieved by UE $k$ as

$$
\begin{aligned}
\hat{R}_{k}= & \left(1-\frac{L_{T}}{L_{E}}\right) \\
& \times \log _{2} \operatorname{det}\left(\boldsymbol{I}_{N}+\hat{\boldsymbol{H}}_{k}^{(c)} \boldsymbol{G}_{k}^{(c)} \operatorname{diag}\left(\boldsymbol{P}_{k}\right) \boldsymbol{G}_{k}^{(c) H} \hat{\boldsymbol{H}}_{k}^{(c) H} \hat{\boldsymbol{\Psi}}_{k}^{(c)-1}\right) .
\end{aligned}
$$

Note that the estimate of the rate achieved by UE $k$ in (15) is computed by assuming that the multiple receive antennas are exploited by performing SIC with IRC ([26], Ch. 10): the rank $l_{k}$ allocated to UE $k$ is given by the number of columns of precoder $\boldsymbol{G}_{k}^{(c)}$, whereas the remaining degrees of freedom are used to partially suppress the residual ICI by IRC. Then, the weighted sum rate $\hat{R}^{(c)}$ is estimated at the CU by solving the following optimization problem:

$$
\hat{R}^{(c)}=\max _{\mathcal{S}_{c} \subseteq \mathcal{U}_{c},\left\{G_{k}^{(c)}\right\}_{k \in \mathcal{S}_{c}}\left\{\boldsymbol{P}_{k}\right\}_{k \in \mathcal{S}_{c}}} \sum_{k \in \mathcal{S}_{c}} \alpha_{k} \hat{R}_{k}
$$

s.t.

$$
\sum_{k \in \mathcal{S}_{c}} \operatorname{tr}\left(\boldsymbol{G}_{k, j}^{H} \boldsymbol{G}_{k, j} \operatorname{diag}\left(\boldsymbol{P}_{k}\right)\right) \leq P^{(\mathrm{BS})}, \quad j \in \mathcal{J}_{c},
$$

where scaling factor $\alpha_{k}$ in (16a) represents the quality of service (QoS) for UE $k$ which depends on the employed scheduler.

Maximization (16) is a well-studied multi-user MIMO problem [29] involving (a) UE selection, (b) transmission rank selection, (c) precoding design, and (d) power allocation. 


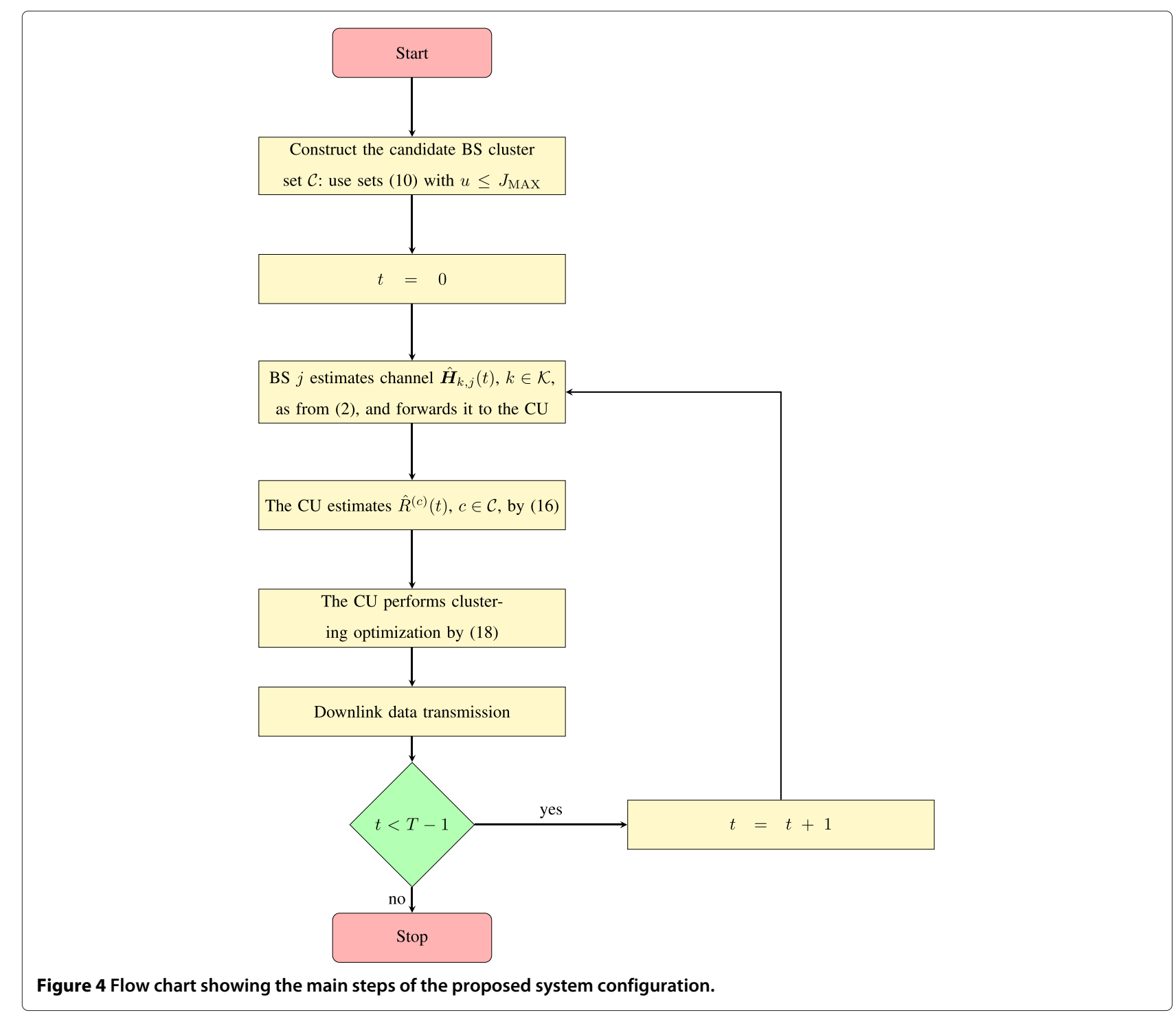

We solve problem (16) by enforcing the assumptions of equal power allocation among the streams sent within cluster $\mathcal{J}_{c}$ and MET introduced in Section 2.2. Moreover, the eigenmodes (and accordingly the set $\mathcal{S}_{c}$ of scheduled UEs and the transmission rank allocated to each UE $k \in \mathcal{S}_{c}$ ) are selected by using a greedy iterative algorithm which, at each iteration, includes the eigenmode which maximizes the weighted sum rate $\hat{R}^{(c)}$ among the ones not scheduled in the previous iterations. The algorithm starts with no UE scheduled and stops when no increase in the weighted sum rate $\hat{R}^{(c)}$ is observed. Cluster $\mathcal{J}_{c}$, among the $N\left|\mathcal{U}_{c}\right|$ possible eigenmodes, selects a maximum of $M\left|\mathcal{J}_{c}\right|$ eigenmodes, due to the limited number of BS antennas. Note that the considered method flexibly adapts to the channel conditions by allowing the allocation of (a) different ranks to different UEs in the same block and (b) different ranks to the same UE across successive blocks.

\subsection{Clustering optimization}

After computing all the weighted sum rates $\hat{R}^{(c)}, c \in \mathcal{C}$, the $\mathrm{CU}$ schedules the set of non-overlapping clusters that maximizes the system weighted sum rate. In detail, by defining

$$
\begin{aligned}
a_{j, c} & = \begin{cases}1, & j \in \mathcal{J}_{c} \\
0, & \text { otherwise }\end{cases} \\
x_{c} & = \begin{cases}1, & \text { CU schedules candidate cluster } \mathcal{J}_{c}, \\
0, & \text { otherwise }\end{cases}
\end{aligned}
$$

we consider that each BS belongs to at most one cluster, i.e., we impose

$$
\sum_{c \in \mathcal{C}} a_{j, c} x_{c} \leq 1, \quad j \in \mathcal{J}
$$


Therefore, at the $\mathrm{CU}$, the clustering optimization is performed by solving the following linear integer optimization problem

$$
\max _{x_{c}, c \in \mathcal{C}} \sum_{c \in \mathcal{C}} \hat{R}^{(c)} x_{c}
$$

s.t. (17).

Note that (18) differs from the optimization carried out in [15] where the objective function simply depends on the received power measured by the UEs.

Maximization (18) is the optimization version of the set packing problem, which is shown to be NP-hard [30]. Hence, as the exhaustive search is not a viable method to solve (18), we propose a greedy iterative algorithm which is reported in Algorithm 1: the proposed solution basically selects at each iteration the best (in terms of system weighted sum rate) cluster and ends when each BS has been assigned to at least one cluster. In detail, let $\mathcal{C}^{(\mathrm{A})}(n)$ be the set identifying the candidate BS clusters considered at iteration $n$. The algorithm starts by imposing $\mathcal{C}^{(\mathrm{A})}(1) \leftarrow$ $\mathcal{C}$ and ends when $\mathcal{C}^{(\mathrm{A})}(n)=\emptyset$. Note that $\mathcal{C}^{(\mathrm{A})}(n)$ identifies all the candidate clusters that do not overlap with the clusters scheduled in the previous iterations. At iteration $n$, we select cluster $w \in \mathcal{C}^{(\mathrm{A})}(n)$ that maximizes the per-BS weighted sum rate, i.e.,

$$
\omega=\underset{c \in \mathcal{C}^{(A)}(n)}{\operatorname{argmax}} \frac{\hat{R}^{(c)}}{\left|\mathcal{J}_{c}\right|}
$$

and we remove from $\mathcal{C}^{(\mathrm{A})}(n)$ all the indexes identifying clusters that partially overlap with $\mathcal{J}_{w}$. Note that in criterion (19), we normalize the cluster weighted sum rate $\hat{R}^{(c)}$ to the number of BSs $\left|\mathcal{J}_{c}\right|$ included in the cluster with the aim of scheduling big clusters only if this really provides an improvement. Let us consider as an example a basic scenario with $J=2$ : by using (19), we schedule the cluster of two BSs only if its weighted sum rate $\hat{R}^{(c)}$ is higher than that achieved when the two BSs are uncoordinated (SCP scheme).

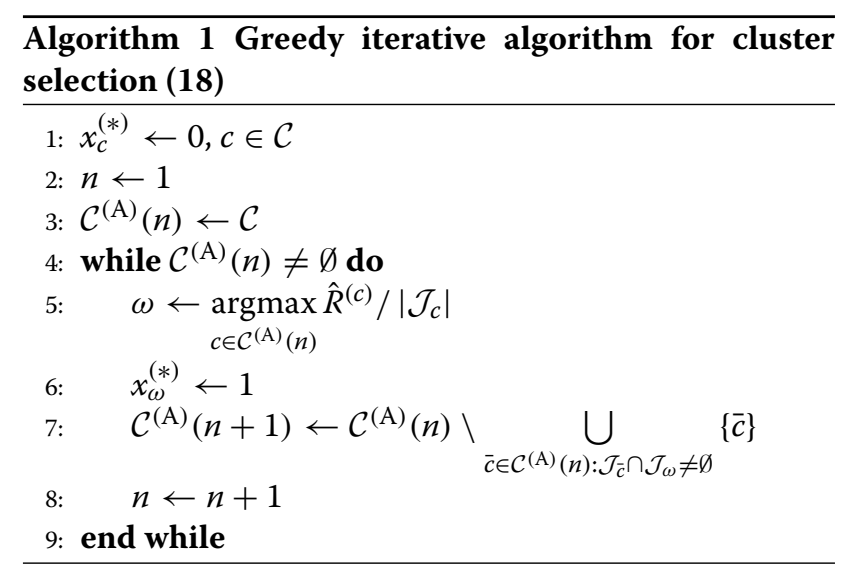

By denoting with $x_{c}^{(*)}$ the greedy solution to (18) obtained by applying the proposed algorithm, the set $\mathcal{S}$ of UEs scheduled in the current block turns out to be

$$
\mathcal{S}=\bigcup_{c \in \mathcal{C}: x_{c}^{(*)}=1} \mathcal{S}_{c}
$$

\section{Numerical results}

We consider a hexagonal cellular scenario where $J=21$ BSs, each equipped with $M=4$ antennas, are organized in seven sites, each with three co-located BSs (see also Figure 1). We consider ten UEs randomly dropped in the coverage area of each BS, with $K=210$ UEs overall. The power available at each $\mathrm{BS}$ is $P^{(\mathrm{BS})}=46 \mathrm{dBm}$, the power available at each $\mathrm{UE}$ is $P^{(\mathrm{UE})}=23 \mathrm{dBm}$, and the thermal noise power is $\sigma_{n}^{2}=-101 \mathrm{dBm}$. The large scale fading between BS $j$ and UE $k$ can be written as

$$
\sigma_{k, j}^{2}=\Gamma^{(\mathrm{CE})}\left(\frac{d^{(\mathrm{CE})}}{d_{k, j}}\right)^{v} e^{\zeta k, j} A\left(\theta_{k, j}\right),
$$

where $d_{k, j}$ is the distance between BS $j$ and UE $k, v=3.5$ is the path loss coefficient, $\left.\Gamma^{(\mathrm{CE})}\right|_{\mathrm{dB}}=10 \mathrm{~dB}$ is the average SNR when an UE is at the cell edge, $e^{\zeta_{k, j}}$ is the lognormal shadowing with $8 \mathrm{~dB}$ as standard deviation, and $A\left(\theta_{k, j}\right)$ models the antenna gain as a function of the direction $\theta_{k, j}$ of $\mathrm{UE} k$ with respect to the antennas of $\mathrm{BS} j$, with

$$
\left.A\left(\theta_{k, j}\right)\right|_{\mathrm{dB}}=-\min \left\{12\left(\theta_{k, j} / \theta_{3 \mathrm{~dB}}\right)^{2}, A_{s}\right\},
$$

where $\theta_{3 d B}=(70 / 180) \pi$ and $\left.A_{s}\right|_{\mathrm{dB}}=20 \mathrm{~dB}([31]$, (21.3)). We consider an inter-site distance of $500 \mathrm{~m}$ and a minimum distance $d_{\text {min }}=35 \mathrm{~m}$ between BSs and UEs. Wraparound is used to deal with boundary effects [32]. We also assume that channels are correlated by considering the popular Kronecker model [33]. By denoting with $\boldsymbol{R}_{\mathrm{BS}}$ the square correlation matrix of size $M$ at the BS, with $\operatorname{tr}\left(\boldsymbol{R}_{\mathrm{BS}}\right)=M$, and with $\boldsymbol{R}_{\mathrm{UE}}$ the square correlation matrix of size $N$ at the UE, with $\operatorname{tr}\left(\boldsymbol{R}_{\mathrm{UE}}\right)=N$, we can write

$$
\boldsymbol{H}_{k, j}(t)=\boldsymbol{R}_{\mathrm{UE}}^{1 / 2} \overline{\boldsymbol{H}}_{k, j}(t)\left(\boldsymbol{R}_{\mathrm{BS}}^{1 / 2}\right)^{H},
$$

where $\overline{\boldsymbol{H}}_{k, j}(t)$ is a matrix of size $N \times M$ whose entries are independent and identically distributed zero-mean complex Gaussian random variables with $\sigma_{k, j}^{2}$ as statistical power.

Results are obtained by simulating 100 UE drops and $T=200$ block channel realizations for each UE drop. We assume that proportional fair scheduling [34] is implemented to provide fairness among UEs, i.e., $\alpha_{k}(t)=$ $1 / \tilde{R}_{k}(t)$, with $\tilde{R}_{k}(t+1)=(1-\gamma) \tilde{R}_{k}(t)+\gamma R_{k}(t), t=$ $0,1, \ldots, T-1$, where $\gamma=0.1$ is the forgetting factor and we initialize $\tilde{R}_{k}(0)=\log _{2}\left(1+P^{(\mathrm{BS})} \sigma_{k, j_{k}}^{2} / \sigma_{n}^{2}\right)$. However, to allow the scheduler to reach a steady state, only the last 
$T / 2$ channels of each UE drop are considered for system performance evaluation.

We compare the developed scheme based on dynamic clustering (DC) against the three static schemes SCP, ISC, and SC, introduced in Section 2. Moreover, we assume that UE scheduling, beamforming design, transmission rank selection, and power allocation are performed, as described in Sections 2.2 and 3.1, also for the static schemes: in particular, UEs are served by using MET [25] with equal power allocation among the eigenmodes and a greedy UE selection is performed within each BS cluster.

For performance evaluation, we assume perfect CSI at the UE side, which employs IRC with SIC, and perfect detection, i.e., there is no error propagation. Moreover, in Section 4.6, we further provide some numerical results that validate the assumption of perfect CSI at the UE employed in most of the CoMP literature.

From (9), the interference plus noise covariance matrix at the UE can be written as

$$
\boldsymbol{\Psi}_{k}(t)=\sigma_{n}^{2} \boldsymbol{I}_{N}+\sum_{m \in \mathcal{S}(t) \backslash\{k\}} \boldsymbol{H}_{k}(t) \boldsymbol{G}_{m}(t) \operatorname{diag}\left(\boldsymbol{P}_{m}(t)\right) \boldsymbol{G}_{m}^{H}(t) \boldsymbol{H}_{k}^{H}(t) .
$$

The effective rate achieved by UE $k$ turns out to be ([26], Ch. 10)

$$
\begin{aligned}
R_{k}(t)= & \left(1-\frac{L_{T}}{L_{E}}\right) \\
& \times \log _{2} \operatorname{det}\left(\boldsymbol{I}_{N}+\boldsymbol{H}_{k}(t) \boldsymbol{G}_{k}(t)\right. \\
& \left.\quad \operatorname{diag}\left(\boldsymbol{P}_{k}(t)\right) \boldsymbol{G}_{k}^{H}(t) \boldsymbol{H}_{k}^{H}(t) \boldsymbol{\Psi}_{k}^{-1}(t)\right) .
\end{aligned}
$$

In (25), the overhead due to the UE pilot transmission is taken into account in the scaling factor before the logarithm.

The proposed schemes are compared in terms of:

- UE rate, defined as

$$
\bar{R}_{k}=\frac{2}{T} \sum_{t=T / 2}^{T-1} R_{k}(t)
$$

- Average cell rate, defined as

$$
\bar{R}_{\text {cell }}=\frac{1}{J} \sum_{k \in \mathcal{K}} \bar{R}_{k}
$$

First, to evaluate the complexity saving achieved by the candidate cluster selection described at the beginning of Section 3, we show in Table 2 the 5 th, the 50th, and the 95th percentiles of the number $|\mathcal{C}|$ of candidate clusters considered with DC by assuming $J_{\mathrm{MAX}}=3$. By adapting the candidate clusters to the long-term channel conditions, we have a saving of about $80 \%$ in terms of $|\mathcal{C}|$ with respect to the full search (11); in fact, with our approach, we ignore candidate clusters that include far apart BSs.
Table 2 Number of candidate clusters with $J_{\text {MAX }}=3$ : comparison between DC and exhaustive search

\begin{tabular}{lcccc}
\hline & DC-5th & DC-50th & DC-95th & Equation (11) \\
\hline$|\mathcal{C}|$ & 235 & 249 & 263 & 1,561 \\
\hline
\end{tabular}

\subsection{Effect of multiple antennas at UEs}

In this section, we consider perfect CSI at BSs, i.e., $\hat{\boldsymbol{H}}_{k, j}(t)=\boldsymbol{H}_{k, j}(t)$ in (2), uncorrelated antennas, i.e., in (23) $\boldsymbol{R}_{\mathrm{BS}}=\boldsymbol{I}_{M}$ and $\boldsymbol{R}_{\mathrm{UE}}=\boldsymbol{I}_{N}$, and we assume $J_{\mathrm{MAX}}=3$ with DC for a fair comparison against ISC and SC in terms of maximum cluster size. In Figures 5 and 6, we report the average cell rate and the fifth percentile of the UE rate for three values of the number $N$ of UE antennas, respectively. First, we observe a significative performance improvement by adding antennas at the UE side. For instance, with SCP by increasing $N$ from 1 to 4 , there is a gain of about $76 \%$ in terms of the fifth percentile of the UE rate. Two factors mainly contribute to this gain: (a) UEs with lower SINR use IRC to limit the impact of residual ICI not managed at the transmit side and (b) UEs with higher SINR can be served by multiple streams of data. From Table 3, where we report the distribution of the number of streams $l_{k}$ with $N=4$, we note that with SCP more than $80 \%$ of the transmissions are rank 1 . On the other hand, with $\mathrm{DC}$, as the interference level suffered by the UEs is lower, about $33 \%$ of transmissions are multi-stream. This shows that in general, most of the gain is due to the IRC and multi-stream transmission plays a non-negligible role only with DC. Then, we observe that ISC provides a moderate gain with respect to SCP in terms of cell rate, but almost no gain in terms of the fifth percentile of the UE rate, whereas the opposite happens with SC. Indeed, ISC of Figure 2, by only allowing cooperation among the sectors of the same site, partially helps the UEs close to the site border, which however get better performance with SC of

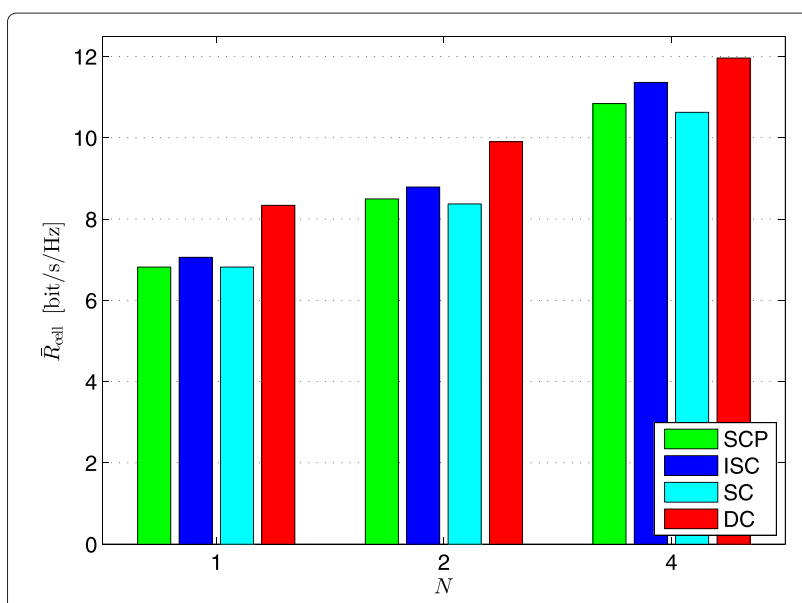

Figure 5 Average cell rate for three values of $N$ and $M=4$. 


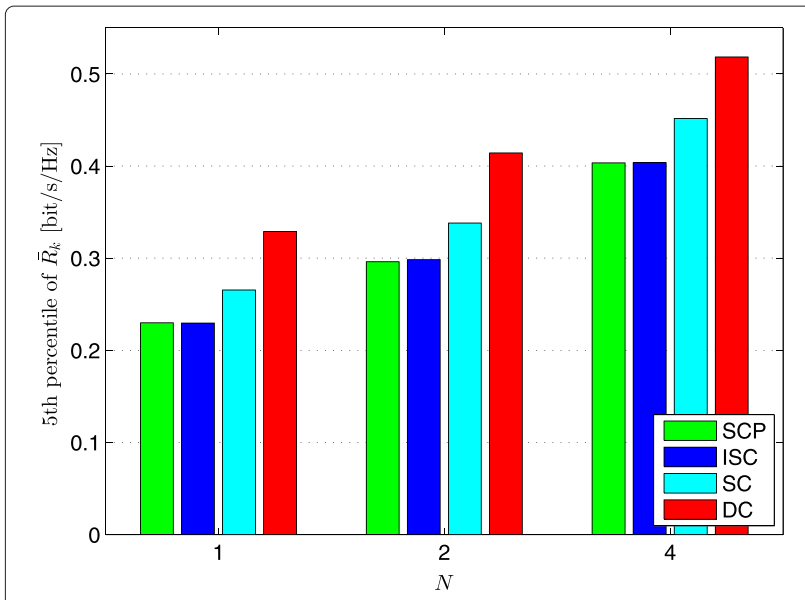

Figure 6 Fifth percentile of the UE rate for three values of $N$ and $M=4$.

Figure 3. Moreover, we also observe that the performance gain achieved by DC over SCP decreases by adding more antennas at the UE side. In fact, as the gain of using multiple antenna UEs is mainly due to the IRC which cancels ICI, the benefits of increasing $N$ are seen more in a noncooperative scenario, where the residual ICI is higher with respect to DC. In detail, from Figure 6, the performance gain achieved by DC over SCP drops from about $43 \%$ with $N=1$ to about $28 \%$ with $N=4$.

To further understand the role of IRC and multi-stream transmission with both SCP and CoMP, we consider now a simplified solution to (16) obtained by assuming a maximum number of streams $l^{\text {(MAX) }}$ that can be transmitted toward each UE and limiting the eigenmodes that can be used for each UE to only the strongest $l^{(\mathrm{MAX})}$ eigenmodes. In Table 4, we report the average cell rate and the fifth percentile of the UE rate by considering $N=4$ and $l^{(\mathrm{MAX})}=1,4$. Note that $l^{\text {(MAX) }}=1$ means that each UE is always served by rank 1 transmissions along its strongest eigenmode and implements IRC, whereas $l^{(\mathrm{MAX})}=N=4$ means that there are no constraints on the number of data streams. Again, as the level of ICI is higher with SCP, in terms of the fifth percentile of the UE rate, the gain achieved by DC over SCP decreases from about $28 \%$ with $l^{(\mathrm{MAX})}=4$ to about $18 \%$ with $l^{(\mathrm{MAX})}=1$. These results confirm that the gain of CoMP with respect

Table 3 Distribution (\%) of $I_{k}$ with $N=4$

\begin{tabular}{lcccc}
\hline & $\boldsymbol{I}_{\boldsymbol{k}}=\mathbf{1}$ & $\boldsymbol{I}_{\boldsymbol{k}}=\mathbf{2}$ & $\boldsymbol{I}_{\boldsymbol{k}}=\mathbf{3}$ & $\boldsymbol{I}_{\boldsymbol{k}}=\mathbf{4}$ \\
\hline SCP & 82.6 & 16.0 & 1.4 & 0.0 \\
ISC & 82.8 & 15.5 & 1.6 & 0.1 \\
SC & 82.9 & 15.7 & 1.4 & 0.0 \\
DC & 67.4 & 21.4 & 8.1 & 3.1 \\
\hline
\end{tabular}

Table 4 Average cell rate and fifth percentile of the UE rate with $N=4$ and $I^{(\text {MAX })}=1,4$

\begin{tabular}{lccccc}
\hline & \multicolumn{2}{c}{$\overline{\boldsymbol{R}}_{\text {cell }}[\mathrm{bit} / \mathbf{s} / \mathrm{Hz}]$} & & \multicolumn{2}{c}{ Fifth percentile of $\overline{\boldsymbol{R}}_{\boldsymbol{k}}[\mathrm{bit} / \mathbf{s} / \mathrm{Hz}]$} \\
\cline { 2 - 3 } \cline { 5 - 6 } $\boldsymbol{I}^{(\mathrm{MAX})}=\mathbf{1}$ & $\boldsymbol{I}^{(\mathrm{MAX})}=\mathbf{4}$ & & $\boldsymbol{I}^{(\mathrm{MAX})}=\mathbf{1}$ & $\boldsymbol{I}^{(\mathrm{MAX})}=\mathbf{4}$ \\
\hline SCP & 10.74 & 10.84 & & 0.415 & 0.404 \\
DC & 11.89 & 11.96 & & 0.491 & 0.518 \\
\hline
\end{tabular}

to the baseline non-cooperative scheme can still be important when UEs are equipped with multiple antennas, but only if multi-stream transmission is properly exploited.

\subsection{Effect of antenna correlation}

In Figure 7 , we consider $N=4, l^{(\mathrm{MAX})}=1$, and perfect CSI at BSs, and we introduce correlation among UE antennas by assuming that $\boldsymbol{R}_{\mathrm{UE}}$ is a symmetric Toeplitz matrix whose first column is $\left[\boldsymbol{R}_{\mathrm{UE}}\right]_{, 0}=\left[1, \beta, \ldots, \beta^{N-1}\right]^{T}$, and plot the fifth percentile of the UE rate in terms of $\beta$. As expected, a higher rate is achieved with low-correlated antennas, i.e., for lower values of $\beta$. Moreover, we also observe that the gain achieved by DC over SCP decreases by decreasing $\beta$ : in detail, this gain drops from $25 \%$ with $\beta=0.9$ to $18 \%$ with $\beta=0.1$. In fact, by decreasing the correlation among UE antennas, we improve the interference suppression capability of IRC. These results confirm that it is not worthy to add more antennas at the UE when they are strongly correlated.

\subsection{Effect of UE selection}

In this section, we consider $N=4, l^{(\mathrm{MAX})}=1$, and perfect CSI at the BSs, and we evaluate how the heuristic greedy UE selection method introduced in Section 3.1 to solve problem (16) and the proposed dynamic clustering scheme impact the gain achieved by CoMP over SCP. Therefore, in Figure 8, we evaluate SCP and DC

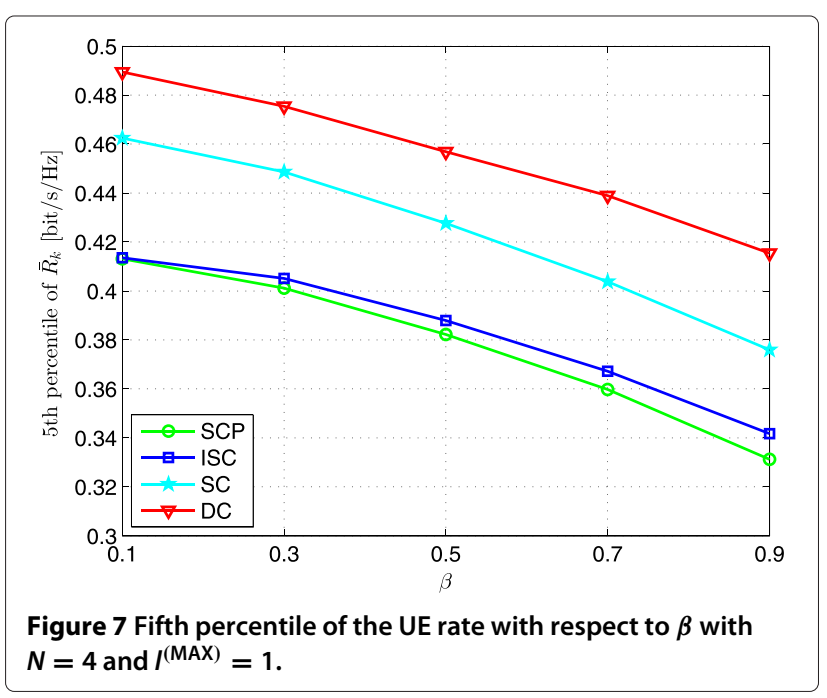




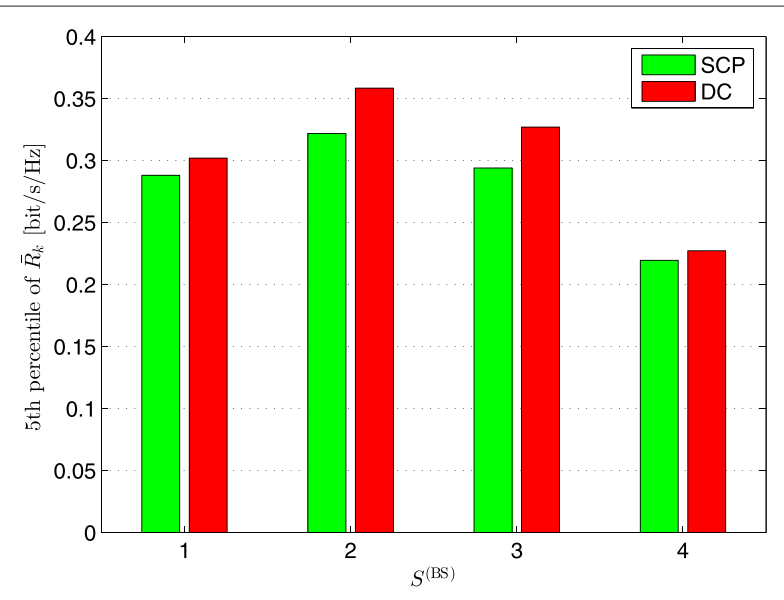

Figure 8 Fifth percentile of the UE rate achieved by a round robin UE selection for four values of $S^{(\mathrm{BS})}$. with $J_{\text {MAX }}=3$ in terms of the fifth percentile of the UE rate by considering a round robin UE selection with $S^{(\mathrm{BS})}=1,2,3,4$ UEs scheduled by each BS: note that when the UEs are selected in a round robin fashion, the gain achieved by CoMP over SCP depends only on the clustering scheme, as no joint optimization of UE scheduling and clustering is performed. First, we observe that $S^{(\mathrm{BS})}=2$ represents the optimal value for both SCP and DC. In fact, when the UEs are scheduled in a round robin fashion, increasing the value of $S^{(\mathrm{BS})}$, while it allows more UEs to be served in each block, it also increases the likelihood of selecting UEs whose channels are almost parallel, thus degrading the achieved rates. Then, when we look at the impact of the UE selection method, we observe that with DC, a gain of about $37 \%$ is achieved by the greedy method over the round robin approach (see also Table 4). Finally, we observe that the gain achieved by DC over SCP decreases from about $18 \%$ with the greedy method to about $11 \%$ with the round robin approach.

\subsection{Effect of cluster size}

In Figure 9 , by assuming $N=1,4, l^{(\mathrm{MAX})}=1$, uncorrelated antennas and perfect CSI at BSs, we compare SCP and DC in terms of the fifth percentile of the UE rate for four values of the maximum cluster size $J_{\text {MAX }}$. An important gain is observed with CoMP by increasing $J_{\mathrm{MAX}}$ : for instance, the gain achieved by DC over SCP increases from $43 \%$ (18\%) with $J_{\text {MAX }}=3$ to about $84 \%$ (40\%) with $J_{\text {MAX }}=6$ when $N=1(N=4)$. These results show that although the strongest interferers are managed by CoMP with $J_{\text {MAX }}=3$, the ICI suffered by UEs is still very high and strongly limits system performance. Hence, a general comment is that BS clusters of higher dimension should be employed if the backhaul infrastructure is able to handle it.

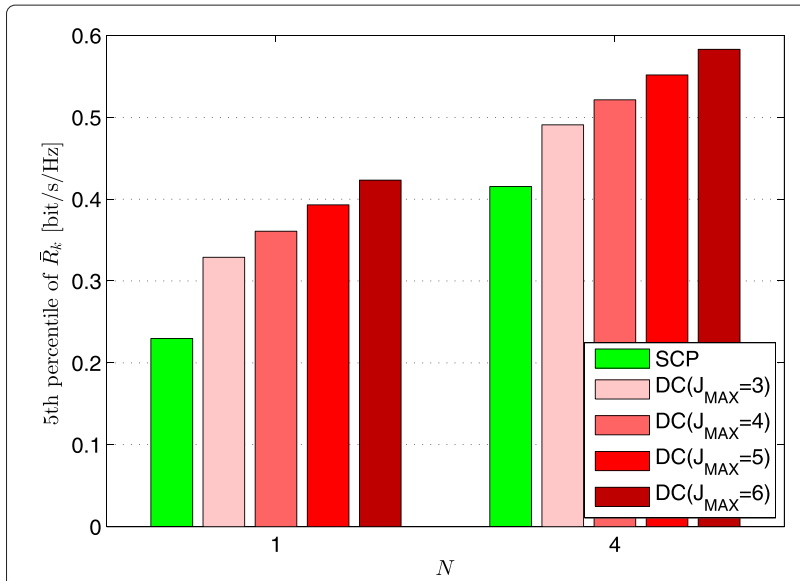

Figure 9 Fifth percentile of the UE rate for two values of $N$ and four values of $J_{\mathrm{MAX}}: I^{(\mathrm{MAX})}=1$.

\subsection{Effect of imperfect CSI at BSs}

In this section, we assume that the CSI at BSs is affected by noise (2) due to the finite number of resource elements $L_{T}$ allocated to the pilot transmissions in each block. After denoting with $f_{\mathrm{d}}$ the maximum Doppler frequency, and with $\bar{\tau}_{\text {rms }}$ the root-mean square delay spread of the channel, we define, respectively, the coherence bandwidth ([35], Ch. 4) and coherence time ([36], Ch. 4) of the channel as

$$
\begin{aligned}
& W_{C}=\frac{1}{\bar{\tau}_{\mathrm{rms}}}, \\
& T_{C}=\frac{0.423}{f_{\mathrm{d}}} .
\end{aligned}
$$

Note that above expressions are only used to determine the block size $L_{E}$ such that the channel can be modeled as uncorrelated between adjacent blocks. Indeed, if $f_{\mathrm{d}}$ or $\bar{\tau}_{\text {rms }}$ increase, $L_{E}$ is reduced and this lowers the rate of each UE as given by (25). Due to the problem of obtaining a reliable CSI at BSs in a high mobility scenario, in the following, we consider $f_{\mathrm{d}}=5 \mathrm{~Hz}$, which at $2.5 \mathrm{GHz}$ carrier frequency roughly corresponds to a mobile velocity of $2 \mathrm{~km} / \mathrm{h}$ ([31], Ch. 21). In this section, we also assume $N=4, l^{(\mathrm{MAX})}=1$, uncorrelated antennas and $J_{\mathrm{MAX}}=3$ with DC.

In Figure 10, we consider the extended pedestrian $\mathrm{A}$ (EPA) model, which is a very low frequency selective channel with $\bar{\tau}_{\text {rms }}=43$ ns ([31], Tab. 21.2). In detail, we show the fifth percentile of the UE rate versus the ratio $L_{T} / L_{E}$, which represents the fraction of resources used for pilot transmission. The dashed lines are the rates computed in Section 4.1 by assuming perfect CSI at BSs. We observe that in this case, rate performance close to the perfect CSI case can be achieved by properly increasing the value of $L_{T}$. Moreover, note that SCP approaches the best performance faster than CoMP schemes. In fact, 


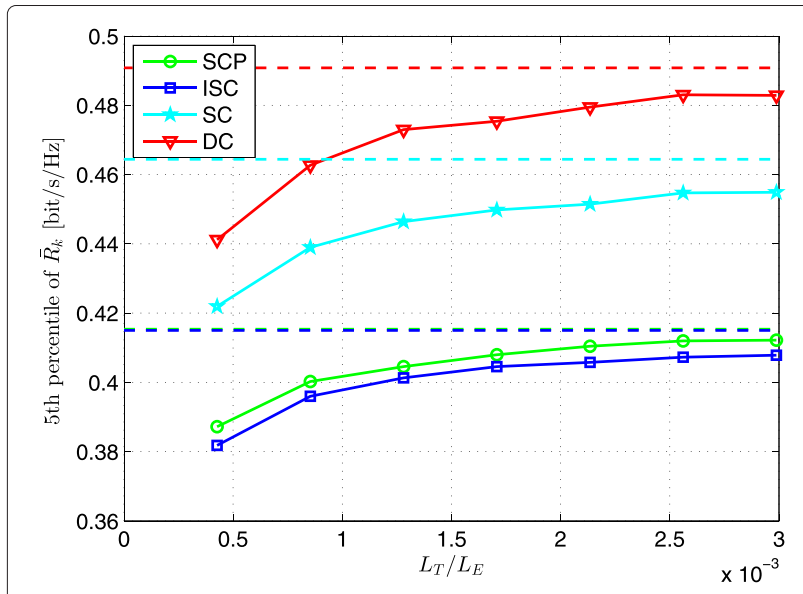

Figure 10 Fifth percentile of the UE rate versus $L_{T} / L_{E}$ with $N=4$, $I^{(\mathrm{MAX})}=1$, and for the EPA channel.

while with SCP only the channels between a BS and its anchored UEs are used for precoding design, with CoMP precoders are optimized on the basis also of the channels between some other auxiliary BSs and these UEs. As these channels are generally characterized by a lower SNR with respect to the channel between a BS and its anchored UEs, more pilots are necessary to collect a reliable CSI at transmit side.

In Figure 11, we plot the fifth percentile of the UE rate versus the ratio $L_{T} / L_{E}$ for the very frequency-selective extended typical urban (ETU) channel model, characterized by $\bar{\tau}_{\mathrm{rms}}=991 \mathrm{~ns}$ ([31], Tab. 21.2). In this case, we observe that rates increase with $L_{T}$ up to a maximum and then decrease. In fact, increasing the value of $L_{T}$ has two conflicting effects: (a) from (3) a more reliable CSI is collected at BSs thus improving performance and (b) a lower

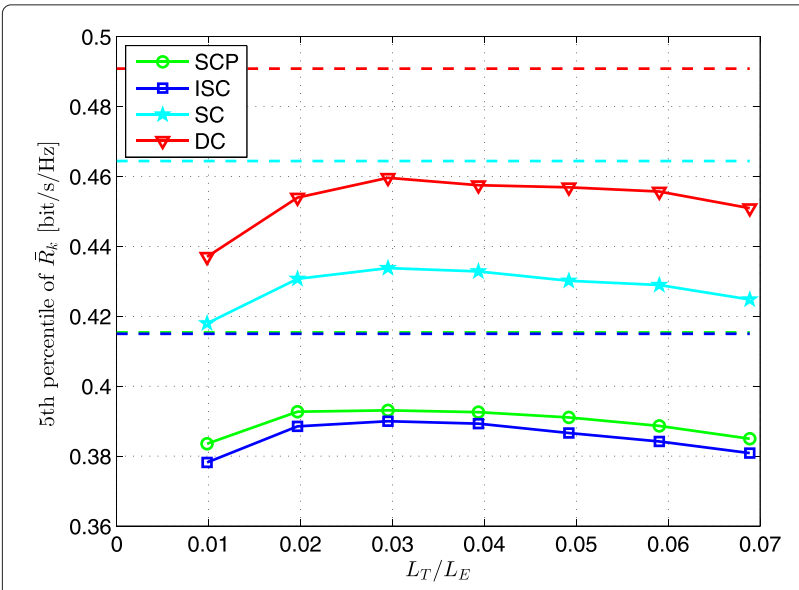

Figure 11 Fifth percentile of the UE rate versus $L_{T} / L_{E}$ with $N=4$, $I^{(\mathrm{MAX})}=1$, and for the ETU channel. number of resource elements is allocated to data transmission thus obviously reducing the achievable rate. Clearly, for lower values of $L_{T}$, the effect of a better CSI dominates, whereas for higher values of $L_{T}$, the CSI is reliable enough for the SINR level of the UEs, and a further increase of the number of pilots represents only a waste of resources. Even if we are still considering a low mobility scenario, due to the higher frequency selectivity of the ETU channel model, no scheme reaches the rates achieved with perfect CSI. Then, as observed for the EPA model, the fraction of resources allocated to pilots necessary to reach the peak in performance is lower for SCP $\left(L_{T} / L_{E} \approx 0.02\right)$ than that for DC $\left(L_{T} / L_{E} \approx 0.03\right)$. By choosing for each scheme the value of $L_{T}$ which provides the best rate, the performance gain achieved by DC over SCP decreases with respect to the perfect CSI case to about $16 \%$.

\subsection{Effect of imperfect CSI at UEs}

In this section, we elaborate on the assumption of perfect CSI at UEs considered in (25). Indeed, it has already been shown $[37,38]$ that in a similar setup, the overhead required to obtain a reliable CSI at UEs is almost negligible when compared to the overhead necessary to acquire CSI at BSs. This result is simply explained by the huge difference in terms of available power at UEs and BSs, which is $23 \mathrm{~dB}$ in a typical LTE scenario: hence, for the same quality in CSI estimate, many more resources are needed for an estimate at BSs than for an estimate at UEs. Moreover, in our model, UE $k$, in order to implement IRC, needs to know the interference covariance matrix $\boldsymbol{\Psi}_{k}(t)$ (24) of size $N \times N$, which also depends on the precoders used by the interfering clusters serving their own UEs. Two estimates of $\boldsymbol{\Psi}_{k}(t)$ at UE $k$ are (a) a simple average of the received samples as proposed in ([39], pag. 10) to directly estimate $\boldsymbol{\Psi}_{k}(t)$ or (b) the use of orthogonal training sequences among different BS clusters to estimate the cascade channel precoders $\boldsymbol{H}_{k}(t) \boldsymbol{G}_{m}(t), m \neq k$, which, in turn, are used to construct matrix $\boldsymbol{\Psi}_{k}(t)$ from (24). Differently from Section 4.5 where the results are shown in terms of the length of the uplink training sequence $L_{T}$, here, we assume perfect CSI at BSs and set the length of the downlink training sequences employed by the clusters to its minimum vale $J M$, equal to the maximum number of streams that can be sent by the BSs. We report in Table 5 the average cell rate and the fifth percentile of the UE rate with SCP and DC by also assuming $N=4, l^{(\mathrm{MAX})}=1$, uncorrelated antennas, and $J_{\operatorname{MAX}}=3$. We consider the

Table 5 Average cell rate and fifth percentile of the UE rate for the ETU channel with imperfect CSI at UEs

\begin{tabular}{lcc}
\hline & $\overline{\boldsymbol{R}}_{\text {cell }}[\mathrm{bit} / \mathrm{s} / \mathrm{Hz}]$ & Fifth percentile of $\overline{\boldsymbol{R}}_{\boldsymbol{k}}[\mathrm{bit} / \mathbf{s} / \mathbf{H z}]$ \\
\hline SCP & 10.69 & 0.414 \\
DC & 11.84 & 0.489 \\
\hline
\end{tabular}


ETU channel in a low mobility scenario with $f_{d}=5 \mathrm{~Hz}$. As expected, when compared to the perfect CSI case reported in Table 4, the performance loss when imperfect CSI is assumed at UEs turns out to be less than 1\%, i.e., almost negligible.

\section{Conclusions}

In this paper, we have considered a downlink CoMP-JP system and, by assuming a maximum cluster size, we have developed a dynamic BS clustering algorithm where the clusters change over time adapting to the channel conditions. We consider that UEs are equipped with multiple antennas that implement IRC and are served by a multistream transmission. The proposed algorithm first defines a set of candidate BS clusters depending on the large scale channel fading. Then, a two-step procedure is applied following a fast fading time scale: (a) first, a weighted sum rate is estimated within each candidate BS cluster by performing UE selection, precoding, power and transmission rank selection, and then (b) the $\mathrm{CU}$ schedules the set of non-overlapping BS clusters that maximizes the estimated system weighted sum rate. Numerical results show that much higher effective rates can be achieved when UEs are equipped with multiple antennas. In fact, by reducing the level of interference suffered by UEs, the proposed approach exploits more the multi-stream transmission than SCP. However, as most of the gain is due to the IRC, the gain achieved by the proposed approach decreases with respect to SCP by increasing the number of UE antennas. Finally, when channel estimation is considered at BSs, the gain promised in the perfect CSI scenario may be achieved only in part; in fact, a better estimate requires a longer training sequence and this lowers the system rate.

\section{Competing interests}

The authors declare that they have no competing interests.

\section{Acknowledgments}

Part of this work has been performed in the framework of the FP7 project ICT-317669 METIS, which is partly funded by the European Union. The authors would like to acknowledge the contributions of their colleagues in METIS, although the views expressed are those of the authors and do not necessarily represent the project. Part of this work has been presented at the International Symposium on Wireless Communication Systems (ISWCS) 2012, Paris (France), and at the International Conference on Signal Processing, Computing and Control (ISPCC) 2013, Shimla (India). This work was carried out when Federico Boccardi was with Bell Labs, Alcatel-Lucent.

\section{Author details}

${ }^{1}$ Bell Labs, Alcatel-Lucent, Lorenzstrasse 10, Stuttgart 70435, Germany. ${ }^{2}$ Vodafone, Newbury, Berkshire RG14 2PZ, UK. ${ }^{3}$ Department of Information Engineering, University of Padova, Via G. Gradenigo 6/b, Padova 35131, Italy.

\section{Received: 13 March 2014 Accepted: 11 July 2014}

Published: 8 August 2014

\section{References}

1. P Marsch, G Fettweis, Coordinated multi-point in mobile communications. (Cambridge University Press, Cambridge, England, 2011)
2. MK Karakayali, GJ Foschini, RA Valenzuela, Network coordination for spectrally efficient communications in cellular systems. IEEE Wireless Commun Mag. 13(4), 56-61 (2006)

3. D Gesbert, S Hanly, H Huang, S Shamai, O Simeone, W Yu, Multi-cell MIMO cooperative networks: a new look at interference. IEEE J. Sel. Areas Commun. 28(9), 1380-1408 (2010)

4. E Björnson, E Jorswieck, Optimal resource allocation in coordinated multi-cell systems. Foundations and Trends in Communications and Information Theory 9(2-3), 113-381 (2012)

5. 3GPP TR 36.819 v11.1.0, Coordinated multi-point operation for LTE physical layer aspects (Release 11), (2011)

6. R Irmer, H Droste, P Marsch, M Grieger, G Fettweis, S Brueck, H-P Mayer, L Thiele, V Jungnickel, Coordinated multipoint: concepts, performance, and field trial results. IEEE Commun. Mag. 49(2), 102-111 (2011)

7. RZakhour, D Gesbert, Optimized data sharing in multicell MIMO with finite backhaul capacity. IEEE Trans. Signal Process. 59(12), 6102-6111 (2011)

8. P Baracca, S Tomasin, N Benvenuto, Constellation quantization in constrained backhaul downlink network MIMO. IEEE Trans. Commun. 60(3), 830-839 (2012)

9. J Zhang, R Chen, JG Andrews, A Ghosh, RW Heath, Networked MIMO with clustered linear precoding. IEEE Trans. Wireless Commun. 8(4), 1910-1921 (2009)

10. A Papadogiannis, D Gesbert, E Hardouin, A dynamic clustering approach in wireless networks with multi-cell cooperative processing, in Proc. IEEE International Conference on Communications (ICC) (Beijing, China, 2008)

11. F Boccardi, H Huang, A Alexiou, Network MIMO with reduced backhaul requirements by MAC coordination, in Proc. IEEE Conference on Signals, Systems and Computers (Asilomar) (Pacific Grove, CA, 2008)

12. J-M Moon, D-H Cho, Inter-cluster interference management based on cell-clustering in network MIMO systems, in Proc. IEEE Vehicular Technology Conference (VTC Spring) (Budapest, Hungary, 2011)

13. J Liu, D Wang, An improved dynamic clustering algorithm for multi-user distributed antenna system, in Proc. IEEE International Conference on Wireless Communications \& Signal Processing (WCSP) (Nanjing, China, 2009)

14. S Zhou, J Gong, Z Niu, Y Jia, P Yang, A decentralized framework for dynamic downlink base station cooperation, in Proc. IEEE Global Communications Conference (GLOBECOM) (Honolulu, HI, 2009)

15. R Weber, A Garavaglia, M Schulist, S Brueck, A Dekorsy, Self-organizing adaptive clustering for cooperative multipoint transmission, in Proc. IEEE Vehicular Technology Conference (VTC Spring) (Budapest, Hungary, 2011)

16. A Papadogiannis, HJ Bang, D Gesbert, E Hardouin, Efficient selective feedback design for multicell cooperative networks. IEEE Trans. Veh. Technol. 60(1), 196-205 (2011)

17. J Gong, S Zhou, Z Niu, L Geng, M Zheng, Joint scheduling and dynamic clustering in downlink cellular networks, in Proc. IEEE Global Communications Conference (GLOBECOM) (Houston, TX, 2011)

18. R Zakhour, D Gesbert, Distributed multicell-MISO precoding using the layered virtual SINR framework. IEEE Trans. Wireless Commun. 9(8), 2444-2448 (2010)

19. M Hong, R Sun, H Baligh, Z-Q Luo, Joint base station clustering and beamformer design for partial coordinated transmission in heterogeneous networks. IEEE J. Sel. Areas Commun. 31(2), 226-240 (2013)

20. F Boccardi, B Clerckx, A Ghosh, E Hardouin, G Jöngren, K Kusume, E Onggosanusi, Y Tang, Multiple-antenna techniques in LTE-advanced. IEEE Commun. Mag. 50(3), 114-121 (2012)

21. I Hwang, C-B Chae, J Lee, RW Heath, Multicell cooperative systems with multiple receive antennas. IEEE Wireless Commun. Mag. 20(1), 50-58 (2013)

22. B Clerckx, H Lee, Y-J Hong, G Kim, A practical cooperative multicell MIMO-OFDMA network based on rank coordination. IEEE Trans. Wireless Commun. 12(4), 1481-1491 (2013)

23. J Winters, Optimum combining in digital mobile radio with cochannel interference. IEEE J. Sel. Areas Commun. 2(4), 528-539 (1984)

24. S Kay, Fundamentals of statistical signal processing, volumel: estimation theory. (Prentice Hall, Upper Saddle River, New Jersey, 1993)

25. F Boccardi, H Huang, A near-optimum technique using linear precoding for the MIMO broadcast channel, in Proc. IEEE International Conference on Acoustics, Speech and Signal Processing (ICASSP) (Honolulu, HI, 2007)

26. D Tse, P Viswanath, Fundamentals of wireless communication. (Cambridge University Press, Cambridge, England, 2005) 
27. P Baracca, F Boccardi, V Braun, A dynamic joint clustering scheduling algorithm for downlink CoMP systems with limited CSI, in Proc. IEEE International Symposium on Wireless Communication Systems (ISWCS) (Paris, France, 2012)

28. H Huh, AM Tulino, G Caire, Network MIMO with linear zero-forcing beamforming: large system analysis, impact of channel estimation, and reduced-complexity scheduling. IEEE Trans. Inf. Theory 58(5), 2911-2934 (2012)

29. QH Spencer, CB Peel, AL Swindlehurst, M Haardt, An introduction to the multi-user MIMO downlink. IEEE Commun. Mag. 42(10), 60-67 (2004)

30. K Hoffman, M Padberg, Set covering, packing and partitioning problems. Springer Encyclopedia of Optimization, 2348-2352 (2001)

31. S Sesia, I Toufik, M Baker, LTE: The UMTS Long Term Evolution. (John Wiley \& Sons, Hoboken, New Jersey, 2009)

32. THytönen, Optimal wrap-around network simulation. Helsinki University of Technology, Report A432 (2001)

33. JP Kermoal, L Schumacher, KI Pedersen, PE Mogensen, F Frederiksen, A stochastic MIMO radio channel model with experimental validation. IEEE J. Sel. Areas Commun. 20(6), 1211-1226 (2002)

34. PViswanath, D Tse, R Laroia, Opportunistic beamforming using dumb antennas. IEEE Trans. Inf. Theory 48(6), 1277-1294 (2002)

35. N Benvenuto, G Cherubini, Algorithms for communications systems and their applications. (John Wiley \& Sons, Hoboken, New Jersey, 2002)

36. T Rappaport, Wireless communications: principles and practice. (Prentice Hall, Upper Saddle River, New Jersey, 2002)

37. T Marzetta, BM Hochwald, Fast transfer of channel state information in wireless systems. IEEE Trans. Signal Process. 54(4), 1268-1278 (2006)

38. KS Gomadam, HC Papadopoulos, C-EW Sundberg, Techniques for multi-user MIMO with two-way training, in Proc. IEEE International Conference on Communications (ICC) (Beijing, China, 2008)

39. J Hoydis, K Hosseini, S ten Brink, M Debbah, Making smart use of excess antennas: massive MIMO, small cells, and TDD. Bell Labs Tech. J. 18(2), 5-21 (2013)

doi:10.1186/1687-1499-2014-125

Cite this article as: Baracca et al:: A dynamic clustering algorithm for downlink CoMP systems with multiple antenna UEs. EURASIP Journal on Wireless Communications and Networking 2014 2014:125.

\section{Submit your manuscript to a SpringerOpen ${ }^{\mathcal{O}}$ journal and benefit from:}

- Convenient online submission

- Rigorous peer review

- Immediate publication on acceptance

- Open access: articles freely available online

- High visibility within the field

- Retaining the copyright to your article

Submit your next manuscript at $\boldsymbol{\wedge}$ springeropen.com 